\title{
AUTOMATED WEED REMOVAL SYSTEM USING CONVOLUTIONAL NEURAL NETWORK
}

\author{
Mounashree J P, N Sanjay, Sushmitha B S, Usha B G \\ Department of Electronics and Communication \\ JSS Science and Technology University (Formerly SJCE) \\ Mysore, Karnataka - 570006, INDIA
}

\begin{abstract}
Weeds are very annoying for farmers and also not very good for the crops. Its existence might damage the growth of the crops. Therefore, weed control is very important for farmers. Farmers need to ensure their agricultural fields are free from weeds for at least once a week, whether they need to spray weed herbicides to their plantation or remove it using tools or manually. The aim of this research is to build an automated weed control system. The system consists of motors, Raspberry pi and a camera which we use to capture the image of the crops and weeds. An automated image classification system has been designed to differentiate between weeds and crops. For the image classification method, we employ the convolutional neural network algorithm to process the image of the object. Deep learning is used to analyze the relevant features from the agricultural images. The dataset is trained for the classification of weed and crop. Therefore, by the use of technology, farmers can reduce the amount of workload and workforce they need to monitor their plantation. In addition, this technology also can improve the quality of the crops.
\end{abstract}

Keywords - Automated Weed Control System, Image classification, Convolutional neural network, Deep learning

\section{INTRODUCTION}

Agriculture is the backbone of Indian economy and village people depend on farming. The profit of plants and vegetables depends on the yield production. One way to get more profit is to remove the weed from the crop. Weeds are an all too common occurrence in lawns and gardens. While some may be deemed useful or attractive, most types of weeds are considered a nuisance. Weeds if not checked may compete with other plantation crops for nutrients, space, water, soil, and restrict their growth. Weeds play host to plant pathogens which infect and degrade the quality of plantations. The conventional method of eliminating weed is to spray herbicides or by manual plucking. The manual weed removal method is a tedious task as it needs huge labour work. Usage of herbicides has an adverse effect on the health of living beings and the surrounding environment. Hence, there is a need to automate the process of weed identification and

\author{
Prof. Mrs. Anupama Shivamurthy \\ Department of Electronics and Communication \\ JSS Science and Technology University (Formerly SJCE) \\ Mysore, Karnataka - 570006, INDIA
}

removal [1]. Thus we propose an approach that aims at designing a system to classify crop and weed. For the weed detection algorithm, the convolutional neural network is suitable for this project because CNN is most commonly applied to analyze visual images.

This paper focuses on detecting the weeds in the crop using convolutional neural network and image processing. Image acquisition is accomplished by the Camera attached to Raspberry pi based Machine learning module. Convolutional Neural Network is used for weed detection using image processing. CNN model is first trained by giving sample images of weed. This CNN model is now deployed onto raspberry pi. Images from camera is sent to raspberry pi at regular intervals. Each captured image is passed onto Trained CNN model for identification of weeds. If it is weed, the area is marked in the original image as weed. In this manner, the weeds are cut using the cutter used in the module.

The rest of the paper is organized as follows. Proposed convolutional neural network algorithms are explained in section II. Experimental results are presented in section III. Concluding remarks are given in section IV.

\section{PROPOSED AlgorithM}

\section{A. Proposed System -}

The proposed system generates the input image by capturing the image by the Rpi camera module. The input image is further processed by image processing to create an image of the area of interest (plants and weeds)[2]. The region of interest is cropped and iteratively input into a pre-trained CNN model to distinguish and classify crops and weeds. The model classifies the input into two categories, namely plants and weeds. The centers of all classified weeds are calculated based on the original reference input image. Later coordinates of the original image are assigned to the original coordinates in the field. Then the cutting machine moves to the appropriate coordinates and removes weeds, and the whole operation repeats the process. 


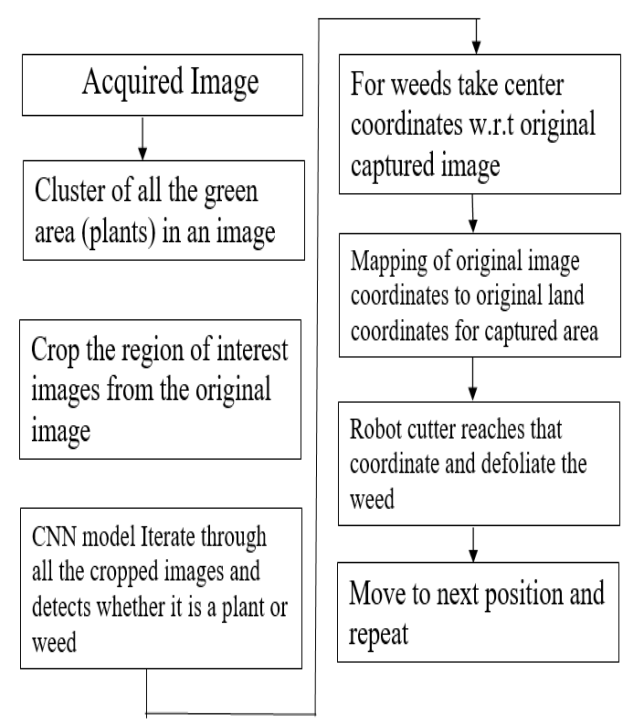

Fig. 1. High level design of proposed system ( The image is acquired using image processing approach[C].)

\section{B. Collection of Data Set of weed images-}

Successful maize cultivation depends heavily on effective weed control. Weed control during the first six to eight weeks after planting is critical as the weeds compete heavily with the crop for nutrients and water during this time. Annual yield losses occur as a result of weed infestations in cultivated crops. Crop yield losses that are attributable to weeds vary with type of weed, type of crop, and the environmental conditions involved. In general, the power loss can fluctuate between 10 and $100 \%$ depending on the extent of the weed control practiced. Zero yield losses from weeds are seldom experienced. Yield losses occur as a result of weeds affecting plant growth and development. This explains why effective weed control is essential. The prerequisite is the correct identification of the weeds.

The dataset collected contains 224 sample images of crop and weed seedlings. The images are grouped into 12 classes. These classes represent common plant species in agriculture. Each class contains RGB images that show plants at different growth stages. The images are in various sizes and are in PNG format.

\section{Image Processing Approach-}

1) Data Preparation: The image is given to the contrast enhancement that is to multiply each pixel value by 0.9 factor. After that, Gaussian noise is added to the image. Then, noise is removed using a Gaussian filter. Applied the excess green method to extract the green part of an image using equation 1 . With the help of the green part, the soil and plants are recognized. Then it is converted into a binary image using otsu's thresholding. It tries to find a threshold value ' $t$ ' that minimizes the weighted within-class variance[4].

$$
\mathrm{Y}=2 \mathrm{G}-\mathrm{R}-\mathrm{B}
$$

Where $\mathrm{R}, \mathrm{G}, \mathrm{B}$ represent the color of an image.

$$
\begin{gathered}
\sigma_{w}^{2}(t)=W_{0}(t) \sigma_{0}^{2}(t)+W_{1}(t) \sigma_{1}^{2}(t) \\
W_{\mathrm{o}}(t)=\sum_{i=0}^{t-1} p(i), W_{1}(t)=\sum_{i=0}^{L-1} p(i)
\end{gathered}
$$

Where, weights W0 (t) and W1 (t) are probabilities of the two classes separated by a threshold $\mathrm{t}, \sigma 20(\mathrm{t})$ and $\sigma 21(\mathrm{t})$ are variances of these two classes, and $\mathrm{L}$ represents the number of bins in histogram.

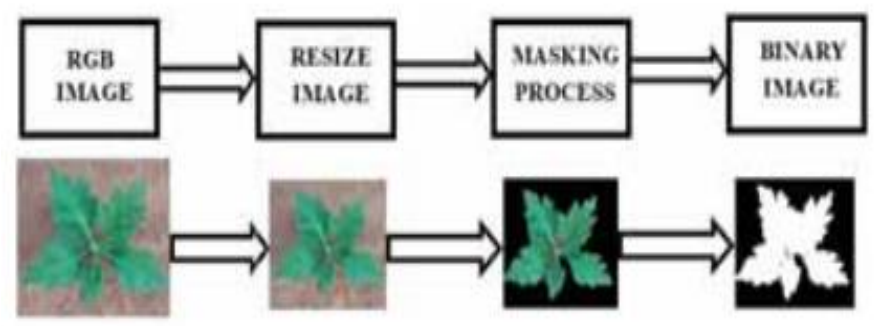

Fig. 2. Pre-processed image

2) Feature Extraction: The shape characters are different for different kinds of crops. Based on the shape features the weed is differentiated from the crop[6]. Here to extract the features, area, perimeter, Eccentricity are calculated. The area is considered for finding the number of white pixels in a binary image. With the help of counter features, the bounding box is drawn to find perimeter, eccentricity. Here the monocotyledon weed is similar to some of the crops. Eccentricity is determined using equation 4.

$$
\text { Eccentricity }=\sqrt{ }\left(1-(\mathrm{b} / \mathrm{a})^{\wedge} 2\right)
$$

Where ' $a$ ' is major axis length and where ' $b$ ' is minor axis length.

\section{Training Convolutional Neural Network-}

The convolutional neural network is the one way to do image recognition, image classification, and detection. Four main layers are used for classification [7][8][9]. In CNN, the trained images and test images are given as input to the convolutional layer. Here, the image features are extracted with the help of the kernel matrix. The different filters are used to find edge detection, blurring of image. Each pixel of an image is multiplied by the filter matrix with the given stride number. The stride is used for a number of shifts over the input matrix. Then a ReLU function is used to introduce the Non-Linearity of an image. It used to convert the negative value to zero for reducing the over fitting problem. After that, a pooling layer is 
used to reduce the dimensionality of the matrix. Here, maxpooling is used to take the largest element in the matrix to rectify the feature map. Finally, a fully connected layer with some activation function like sigmoid and softmax is used, to give some probability value for an image. Based on that probability the classification is done. In this work, the CNN is used with a convolutional layer with ReLU function to extract the features from an image. Max pooling is used to reduce the size of the matrix to avoid an over-fitting problem. Finally, the fully connected layer is used to multiply the input matrix to the sigmoid function for the classification [5][10].

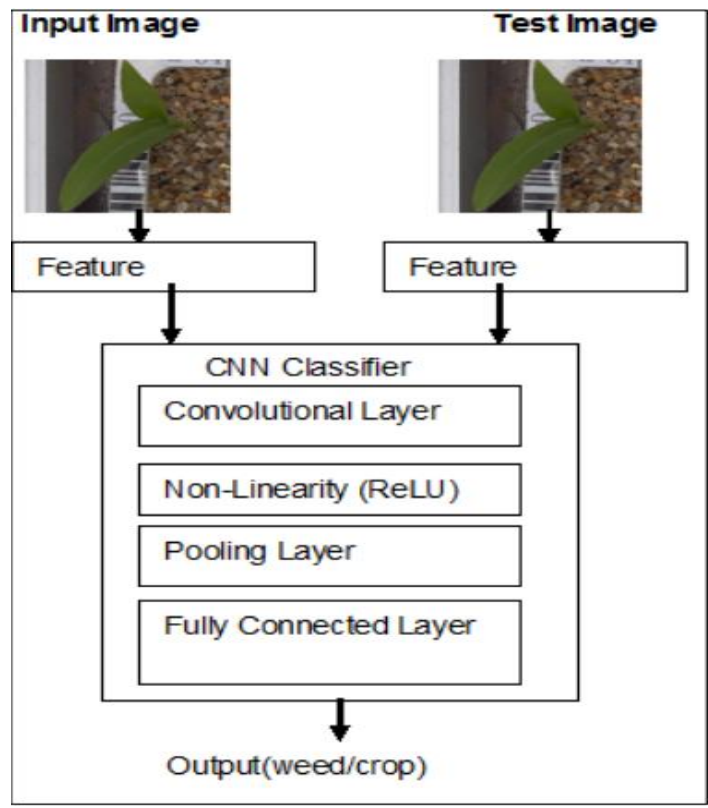

Fig. 3. Classification model

\section{E. Architecture of the proposed system -}

Once the CNN network is trained it has to be deployed on Raspberry pi. Training the CNN network on raspberry pi takes a huge time since RAM is very less. Hence CNN network is trained on laptop and then the file which contains weights of the neural network is deployed on raspberry pi. CNN network classifies each segment crop. If it's a weed the segment is marked in the original image as weed then the cutter moves to the corresponding coordinate and defoliates the weed and the whole operation iterates for the whole farm [3].

Weed and crop classification is used for the identification of weed and helps to automate the weed removal process.

The system works with the uniform land, Non overlapping and vertical alignment condition of weed and crop.

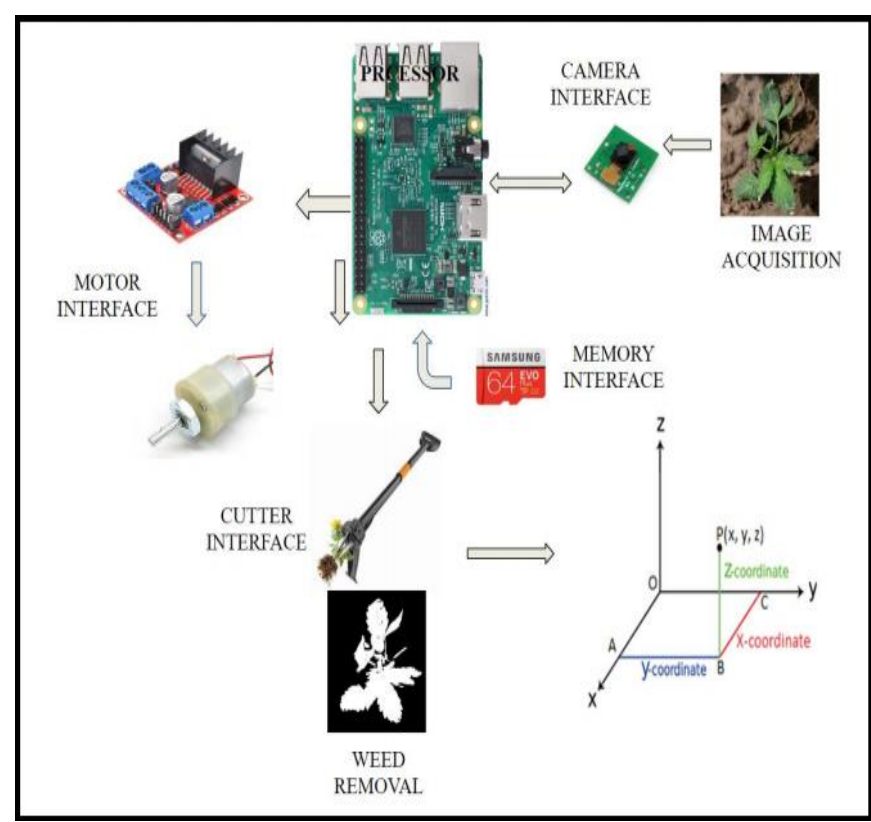

Fig. 4. Block Diagram of the system

\section{EXPERIMENT AND RESULT}

Python programming language is used for implementation of this work. The experiment is run on Python 3.6 IDLE with the help of OpenCV, Keras and Tensorflow packages. Keras and Tensorflow is an important package to create a neural network and help develop this framework in deep learning. The proposed system uses CNN for identifying weeds. We have used 12 varieties of weeds which contain 224 sample images for training $\mathrm{CNN}$ network and it produced an average accuracy of $98 \%$.

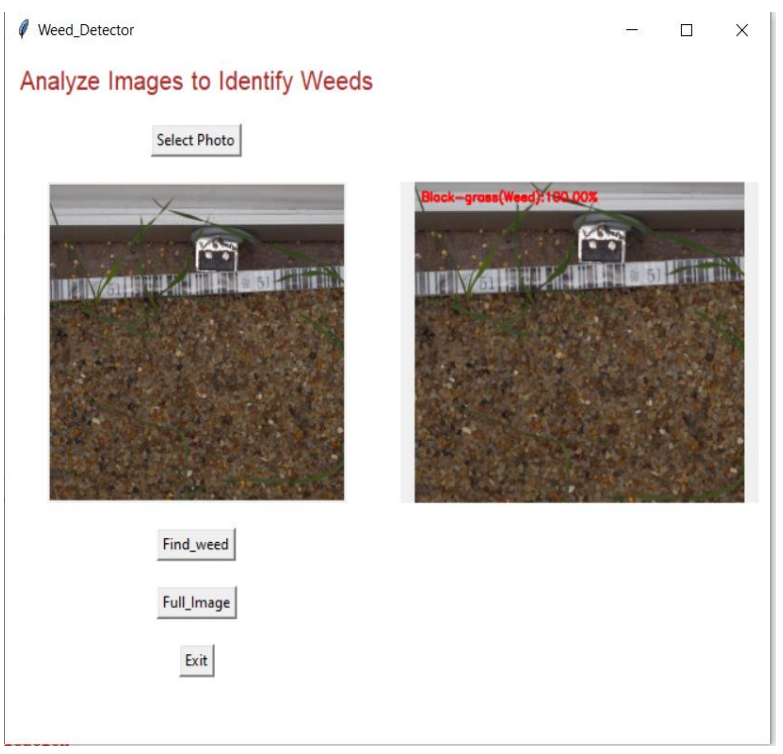

Fig. 5. The image of detected Black-grass weed 


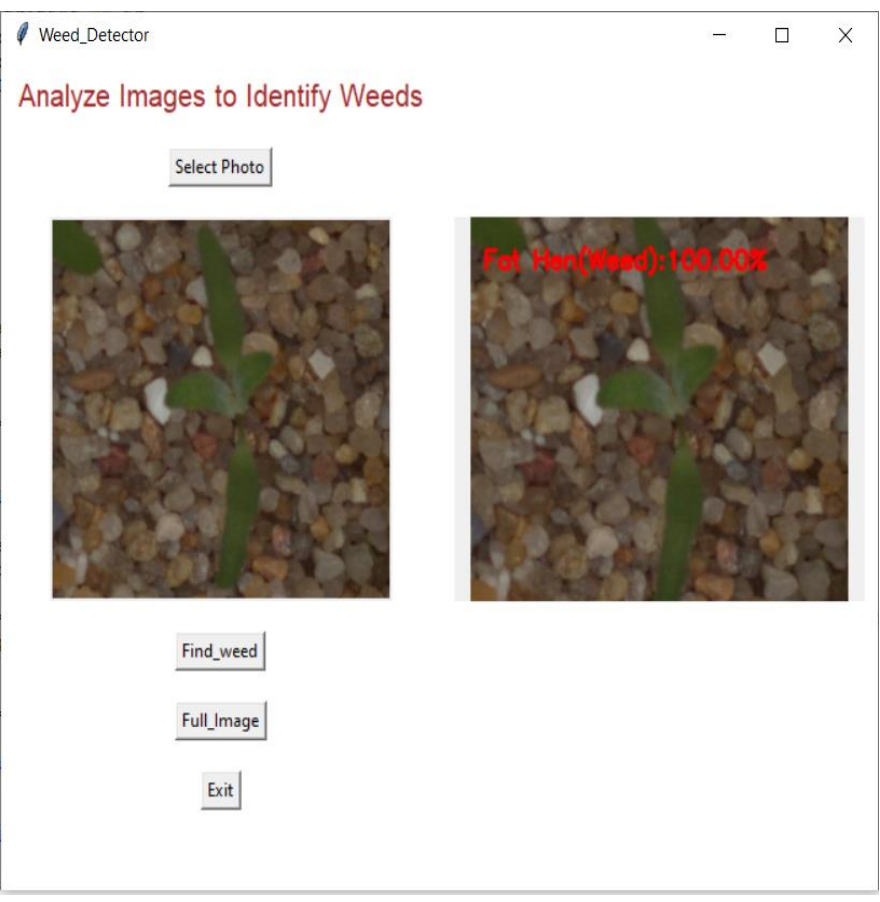

Fig. 6. The image of Detected Fat Hen weed

The proposed scheme is tested using the system. From the simulation of the experiment results, we can draw to the conclusion that this method gives $98 \%$ accuracy.

Table 1 - Experiment Result

\begin{tabular}{|l|l|l|}
\hline $\begin{array}{l}\text { Sl. } \\
\text { No. }\end{array}$ & Images tested with the system & Accuracy \\
\hline 1 & Black-grass (weed) & $\mathbf{1 0 0 \%}$ \\
\hline 2 & Charlock (weed) & $\mathbf{9 9 \%}$ \\
\hline 3 & Cleavers (weed) & $\mathbf{9 8 . 9 \%}$ \\
\hline 4 & Common Chickweed (weed) & $\mathbf{9 9 . 8 \%}$ \\
\hline 5 & Fat Hen (weed) & $\mathbf{1 0 0 \%}$ \\
\hline 6 & Loose-Silky-bent (weed) & $\mathbf{9 9 \%}$ \\
\hline 7 & Scentless Mayweed (weed) & $\mathbf{9 9 . 9 \%}$ \\
\hline 8 & Shepherds-Purse (weed) & $\mathbf{9 8 . 8 \%}$ \\
\hline 9 & Small-flowered-Cranesbill (weed) & $\mathbf{1 0 0 \%}$ \\
\hline
\end{tabular}

Table 1 shows the obtained results for the proposed system.

\section{CONCLUSION}

Weed control and detection has become one of the most interesting domains. Preventing the use of chemicals as well as saving money are some reasons of this trend. The proposed system, automated Weed detection using CNN and Image processing uses a Convolutional Neural network for detecting weeds. The classification of weed and crop is used to detect the weed and remove it by using an automated cutter to improve the productivity of the crop. $\mathrm{CNN}$ is used to extract the more relevant features of an image. The future work is to develop an automatic process.

\section{REFERENCE}

[1] Alexander Wendel and James Underwood,SelfSupervised Weed Detection in Vegetable Crops Using Ground Based Hyperspectral Imaging",IEEE International Conference on Robotics and Automation (ICRA), Sweden, pp. 16-21, May 2016.

[2] Ukrit Watchareeruetai, Yoshinori Takeuchi, Tetsuya Matsumoto, Hiroaki Kudo, Noboru Ohnishi,/Computer Vision Based Methods for Detecting Weeds in Lawns", IEEE Journal of Agricultural Engineering Research, vol. 78, no. 3, pp. 233-243, 2001.

[3] Amir H. Kargar B., Ali M. Shirzadifar, \Automatic Weed Detection System and Smart Herbicide Sprayer Robot for corn fields," IEEE International Conference on Robotics and Mechatronics, Tehran, Iran, pp. 468-473, February 2013.

[4] Su Chen and Robert M. Haralick, "Recursive Erosion, Dilation,Opening, and Closing Transforms," IEEE Transactions on Image Processing, vol. 4, no. 3, pp. 335345, March 1995.

[5] Mohd. Marzuki Mustafa, Aini Hussain, Kamarul Hawari Ghazali and Slamet Riyad, "Implementation of Image Processing Technique in Real Time Vision System for Automatic Weeding Strategy," IEEE International Symposium on Signal Processing and Information Technology, pp. 632-635, December 2007.

[6] A. Sokolowski and T. Pardela, "Fourier transforms in melanoma image classification," International Conference on Human System Interactions (HSI), pp. 386-389, June 201334 ASTRA 2018-19.

[7] Sebastian Haug, Andreas Michaels, Peter Biber and Jorn Ostermann, "Weed Identification Method based on Probabilistic Neural Network in the Corn Seedlings Field," International Conference on Machine Learning and Cybernetics, pp. 1528-1531, 2010.

[8] Jiazhi Pan, Min Huang and Yong He, "Crop and Weed Image Recognition by Morphological Operations and ANN model," Instrumentation and Measurement Technology Conference(IMTC), pp. 1-4, 2007.

[9] Philipp Lottes, Jens Behley, Andres Milioto and Cyrill Stachniss, "Fully Convolutional Networks with Sequential Information for Robust Crop and Weed Detection in Precision Farming," IEEE Robotics and Automation Letters, vol.3, no.4, pp. 2870- 2877, October 2018. 
International Journal of Engineering Applied Sciences and Technology, 2021

Vol. 6, Issue 2, ISSN No. 2455-2143, Pages 231-235

Published Online June 2021 in IJEAST (http://www.ijeast.com)

[10] Abdul Kadir, Lukito Edi Nugroho, Adhi Susanto and Paulus Insap Santosa, "Leaf Classification Using Shape, Color and Texture Features," IEEE International Symposium on Signal Processing and Information Technology, pp. 225-230, 2015. 